\title{
Review Article \\ Single Top as Window on Heavy Quarks and Other New Physics
}

\author{
Andrea Giammanco ${ }^{1,2,3}$ \\ ${ }^{1}$ Centre for Cosmology, Particle Physics and Phenomenology-CP3, Université Catholique de Louvain, \\ Chemin du Cyclotron, 2, B-1348 Louvain-la-Neuve, Belgium \\ ${ }^{2}$ Fonds National de la Recherche Scientifique (FNRS), Rue d'Egmont 5, B-1000 Brussels, Belgium \\ ${ }^{3}$ National Institute of Chemical Physics and Biophysics, Akadeemia tee 23, 12618 Tallinn, Estonia \\ Correspondence should be addressed to Andrea Giammanco; andrea.giammanco@cern.ch
}

Received 22 June 2012; Accepted 4 December 2012

Academic Editor: Kai-Feng Chen

Copyright (c) 2013 Andrea Giammanco. This is an open access article distributed under the Creative Commons Attribution License, which permits unrestricted use, distribution, and reproduction in any medium, provided the original work is properly cited.

\begin{abstract}
This paper discusses what the study of the electroweak production of single top quarks can tell us about additional quark generations, and how to distinguish the new quarks hypothesis from other kinds of new physics. We also suggest some new studies that become possible for the first time thanks to the large statistics of single top quarks produced at the LHC.
\end{abstract}

\section{Introduction}

The top quark is the heaviest elementary particle discovered so far, and in many ways it is a very uncommon quark. The fact that its electroweak decay is faster than the hadronization timescale implies that the top quark exists only as a free quark, so that the effects from new physics could show up very clearly by comparing measurements with the precise Standard Model (SM) predictions. While the pair-production process $(t \bar{t})$ has already entered several years ago the domain of "precision physics," the study of single top production, started with the discovery at Tevatron in 2009 [1, 2], has been limited so far. The complexity of its final state demands a good control of the experimental apparatus, and other SM processes (including $t \bar{t}$ ) provide an overwhelming background.

The "rediscovery" of single top quark production at the Large Hadron Collider (LHC) with the early $7 \mathrm{TeV}$ data has been a major milestone for the ATLAS [3] and CMS [4] experiments, which are now working towards the ultimate precision and the full coverage of all production channels at $7 \mathrm{TeV}$ and $8 \mathrm{TeV}$. Collisions at the Tevatron and at the LHC give access to different mixtures of electroweak single top processes: $t$-channel and $s$-channel production (Figures 1(a) and $1(\mathrm{~b}))$ contribute less evenly at $7 \mathrm{TeV}(65 \mathrm{pb}$ and $4.6 \mathrm{pb}$, resp.) than at $1.96 \mathrm{TeV}(2 \mathrm{pb}$ and $1 \mathrm{pb})$, and $W$-associated production $(t W$, Figure $1(\mathrm{c}))$ which is virtually inaccessible at the Tevatron becomes the second-largest contributor at the LHC (15 pb). A feature of SM single top production at LHC, absent at Tevatron, is the asymmetry between top and antitop production in $t$-channel ( 43 versus $23 \mathrm{pb}$ at $7 \mathrm{TeV}$ ) and $s$-channel ( 3.1 versus $1.4 \mathrm{pb}$ at $7 \mathrm{TeV}$ ). The first evidence of $t W$ production has been reported by the ATLAS collaboration [5], followed by CMS [6], using $7 \mathrm{TeV}$ data. Both measurements are systematics-limited, and reaching the $5 \sigma$ level will require clever analysis improvements and more precise constraints of the leading systematics (related to $t \bar{t}$ modeling and, in the CMS case, to $b$-tagging efficiency).

ATLAS and CMS recorded more than $5 \mathrm{fb}^{-1}$ of pp collisions delivered by the LHC at $7 \mathrm{TeV}$ during 2011, and the same amount has been reached within a few months of running at $8 \mathrm{TeV}$ in 2012. Significant improvements of the $t$ channel cross-section measurement at $7 \mathrm{TeV}[7,8]$ and the first measurement of the $t$-channel cross-section at $8 \mathrm{TeV}$ $[9,10]$ have been performed by both collaborations. The first $t W$ cross s-ction measurement at $8 \mathrm{TeV}$ is expected very soon. The signal-to-background ratio improves from 7 to $8 \mathrm{TeV}$ for the $t W$ production, whose cross-section is expected to increase by $40 \%$, and for $t$-channel which increases by $35 \%$, against a $20 \%$ increase for inclusive $W$ production and $25 \%$ for the $W Q(\bar{Q})$ process (with $Q=b, c$ ). The $s$-channel cross-section, instead, increases by only $20 \%$. A possibility not yet exploited by the LHC analyses is to take advantage of the different dependencies of these processes on energy, for example, by simultaneous fits to 7 and $8 \mathrm{TeV}$ 


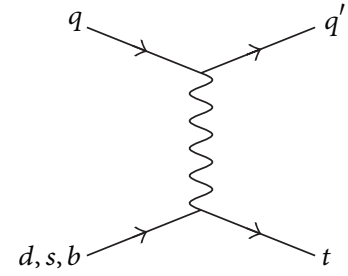

(a)

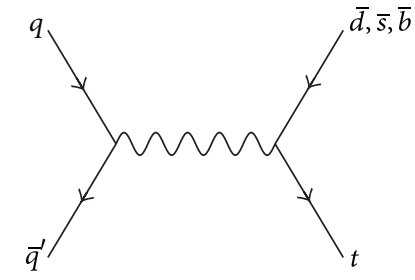

(b)

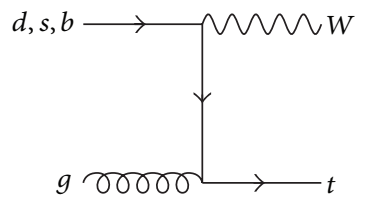

(c)

FIGURE 1: Representative diagrams for single top production in the Standard Model: $t$-channel (a), $s$-channel (b), and $W$-associated production or $t W(\mathrm{c})$.

data with $\sigma_{b k g}(8 \mathrm{TeV}) / \sigma_{b k g}(7 \mathrm{TeV})$ ratios constrained to SM expectations.

Table 1 shows the state of the art for theory predictions and experimental measurements of the cross-section of the three production mechanisms. Figure 2 shows the dependence of the cross-section for $t$-channel production on centre-of-mass energy. This is the only single top process whose cross-section has been measured at three centre-ofmass energies so far.

The rest of this paper elaborates on what the study of the properties of singly produced top quarks can tell us about physics beyond the SM, with particular attention to what it can tell us about new heavy quarks. Section 2 discusses the most straightforward connection between single top studies and new sequential quark generations, which is the extraction of $\left|V_{t b}\right|$ from the single top cross-section under some assumptions, which are critically discussed. Section 3 is devoted to single top polarization, a crucial feature of $t$ channel production in the SM which would hold true also with the inclusion of sequential quark generations in the model and can be a powerful discriminator with respect to other forms of new physics. Section 4 elaborates more generally on which kind of new physics can be suspected to be at play in case of different patterns of deviations from the SM expectations in the phase space sampled by single top analyses, with a bottom-up approach. Finally, Section 5 mentions some single top signatures which are absent in the SM.

\section{Single Top and $\left|V_{t b}\right|$}

Typically, a single top cross-section measurement paper ends with the inference of the $\left|V_{t b}\right|$ interval corresponding to the measured cross-section, under the simplifying assumption that, whatever the values, the relationships $\left|V_{t b}\right| \gg\left|V_{t d}\right|$ and $\left|V_{t b}\right| \gg\left|V_{t s}\right|$ hold true. Also implicit is the assumption of purely left-handed $t b W$ coupling (i.e., left-handed and right-handed helicity fractions $f_{L}=1, f_{R}=0$ ). Table 2 shows the $\left|V_{t b}\right|$ intervals extracted by the Tevatron and LHC experiments under these assumptions, with and without the unitarity constraint $0 \leq\left|V_{t b}\right| \leq 1$. The most precise determination from single top [8] reached a comparable precision with the best one derived from the branching ratio $R_{b} \equiv \Gamma(t \rightarrow W b) / \Gamma(t \rightarrow W q)$, where $q=d, s, b[11]$, which is interpreted as $R_{b}=\left|V_{t b}\right|^{2} /\left(\left|V_{t d}\right|^{2}+\left|V_{t s}\right|^{2}+\left|V_{t b}\right|^{2}\right)$ under the same assumptions (and, therefore, can be used to infer $\left|V_{t b}\right|$ directly only if the additional assumption $\left|V_{t d}\right|^{2}+\left|V_{t s}\right|^{2}+$ $\left|V_{t b}\right|^{2}=1$ is imposed).

Some papers [12-14] examined how single top crosssections are modified under the hypothesis of additional quarks mixing with the known up-type quarks, and therefore of a violation of unitarity for the $3 \times 3$ components of the extended CKM matrix. While the sum $\left|V_{t d}\right|^{2}+\left|V_{t s}\right|^{2}+$ $\left|V_{t b}\right|^{2}+\left|V_{t b^{\prime}}\right|^{2}$ and, a fortiori, the sum $\left|V_{t d}\right|^{2}+\left|V_{t s}\right|^{2}+\left|V_{t b}\right|^{2}$ is bound to be $\leq 1$ also in the extended matrix, the limits on $\left|V_{t d}\right|$ and $\left|V_{t s}\right|$ derived from precision physics under the SM assumption do not hold. Therefore, contrary to common intuition, additional quark generations could result in an enhancement, and not only necessarily a deficit, of the single top cross-section in $t$-channel and $t W$ productions, due to the large parton densities of $d$ and $s$ quarks in the proton (much larger than the $b$ density), causing an amplification of the effect of any $V_{t d}$ or $V_{t s}$ value larger than the SM expectation, which can overcome the deficit due to a smaller $V_{t b}$.

References $[13,14]$ performed the exercise of deriving less model-dependent limits on all the three $\left|V_{t q}\right|$ matrix elements by reexamining the measurements of single top cross-sections and $R_{b}$ published at the time. This typically imposes several approximations and shortcuts, not having access to the raw data. A particularly tricky case for the reinterpretation are single top analyses based on multivariate techniques, because several of the input variables are related to the kinematics of a reconstructed top quark, and the choice itself of the jet that is assumed to come from the decay of the top quark is made under the assumption that it is a $b$ jet, and therefore $\left|V_{t b}\right| \approx 1$. It is, therefore, important that the experimental collaborations carry out such an exercise themselves, with minimal model dependence at every stage of the analysis, possibly training the multivariate discriminators for different $\left|V_{t b}\right|$ scenarios.

\section{What Can Be Learned from Angular Observables}

A crucial property of the top quark is its lifetime which is shorter than the QCD decoherence timescale $(\tau \approx 4 \times$ $10^{-25} \mathrm{~s}$ the former, two orders of magnitude more the latter), causing its decay products to retain memory of its helicity. This provides additional powerful tools in the search for new physics in single top studies: single top production in $t$ - and $s$-channel yields $100 \%$ polarized top quarks if only electroweak interactions are involved (which stays true if the only extension to the SM is a sequential new family of quarks), while different new physics models yield different polarizations. Relevant to this paper is the fact that, in case of hints of new physics in the single top sector, observing or 


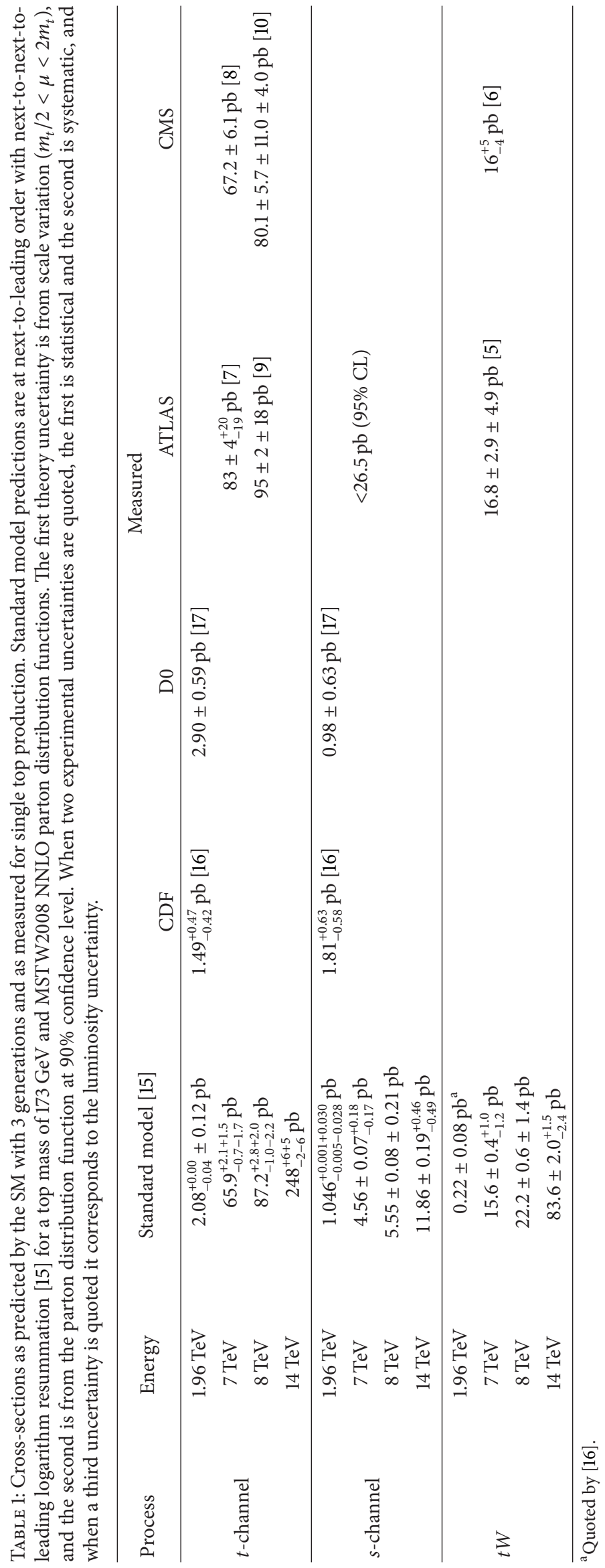




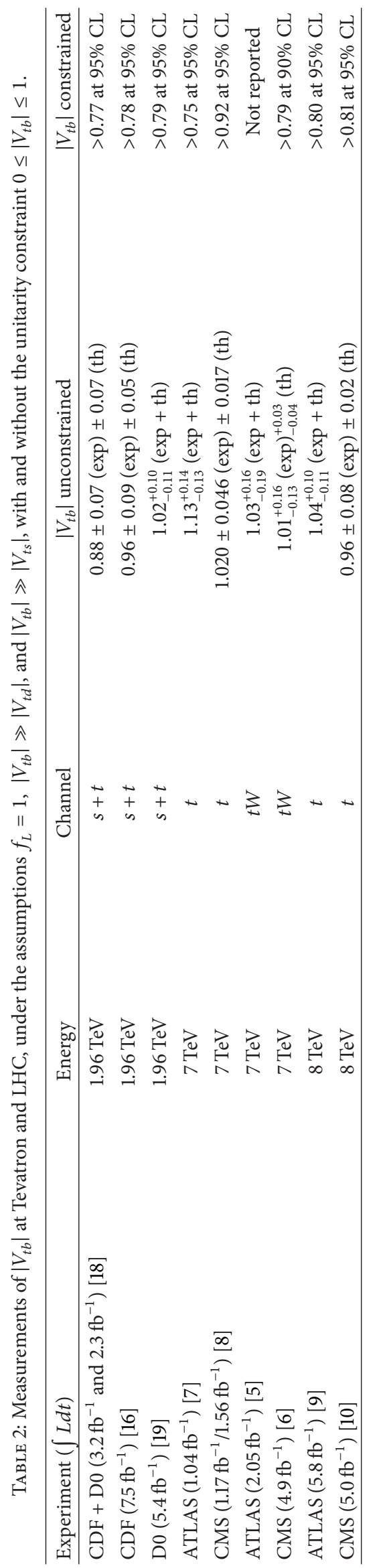




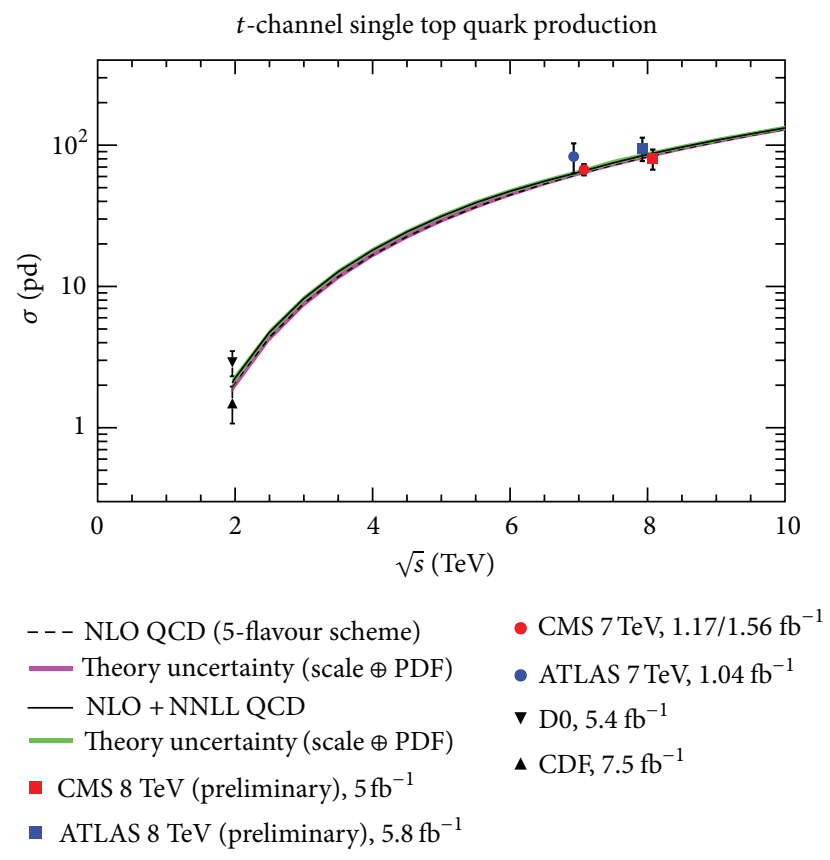

FIGURE 2: Single top cross-section in the $t$-channel versus centre-of-mass energy, comparing the existing measurements at Tevatron and LHC with the QCD expectations computed at NLO with MCFM in the 5-flavour scheme [24] and at NLO + NNLL [25]. The error band (width of the curve) includes the uncertainty on the top mass, the PDF uncertainty estimated according to the HEPDATA recommendations [26], and the factorization and renormalization scales changed coherently by a factor two up and down.

not a SM-like (i.e., close to $100 \%$ ) polarization of the top quark would discriminate between a trivial extension of the SM with additional quarks and other models.

The general form of the angular distribution $\left(\theta_{X}\right)$ of decay product $X(=W, \ell, v, b)$ in the top quark rest frame is

$$
\frac{d \Gamma}{d \cos \theta_{X}}=\frac{\Gamma}{2}\left(1+P_{t} \alpha_{X} \cos \theta_{X}\right),
$$

where $P_{t}$ is the single top polarization (due to the production vertex) along a given direction chosen to quantise the top spin, and $\alpha_{X}$ is a spin-analysing power specific of the decay particle (maximum for the charged lepton [20, 21], which is also preferred in this kind of studies because of the identification cleanness of electrons and muons). Both parameters can be affected by top quark anomalous couplings [20].

Single top polarization in the $t$-channel process is already manifest in the slope of the $\cos \theta_{\ell}^{*}$ distribution (see Figure 3 for a selection of enriched in $t$-channel events, from [8]), where $\theta_{\ell}^{*}$ is defined as the angle between the charged lepton and the light jet $\left(j^{\prime}\right)$ in the reconstructed top quark rest frame. The rationale for this definition is that the (light) quark recoiling against the single top quark tends to have a direction parallel to the spin direction of the top quark at the production vertex [21]. The SM expectation $P_{t} \times \alpha_{\ell} \approx 1$ has even been built-in in the foundations of one of the earliest cross-section measurements at the LHC [22] which made use of the $\cos \theta_{\ell}^{*}$ distribution to fit the SM signal (this is named the "2D analysis" in [22], being based on a likelihood fit to the bidimensional distribution in the $\left(\cos \theta_{\ell}^{*}, \eta_{j^{\prime}}\right)$ plane.

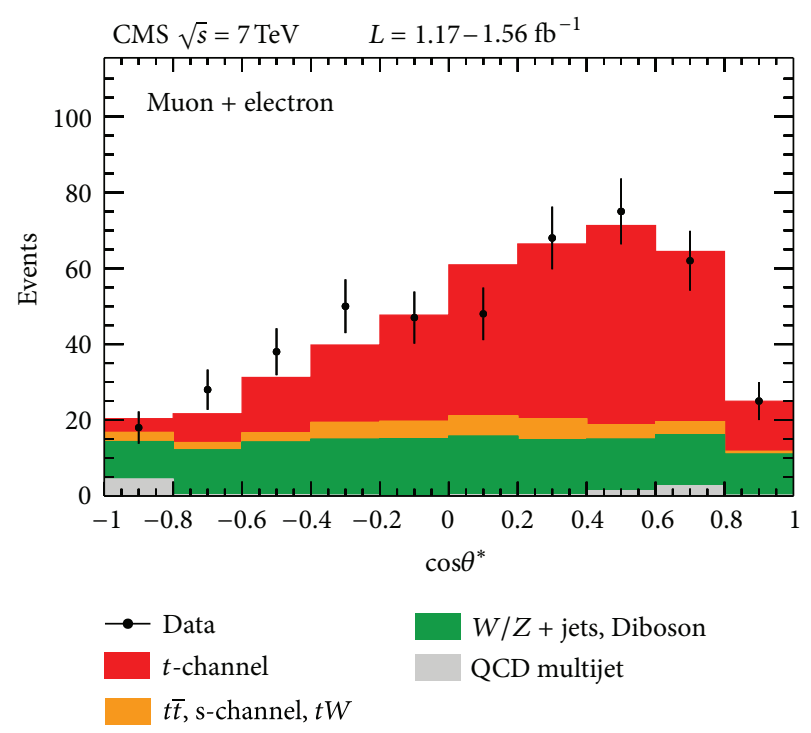

Figure 3: Distribution of $\cos \theta_{\ell}^{*}$ in the $\left|\eta_{j^{\prime}}\right|>2.8$ region, from [8].

In the same paper, this is combined with the result of a fit to a boosted decision trees where $\cos \theta_{\ell}^{*}$ is one among many inputs) and it is part of the implicit assumptions of all the multivariate analyses at Tevatron and LHC. It is crucial to perform unbiased precision measurements of both the cross-section and $P_{t}$ to decouple the issue of measuring the SM-like component from the search for new physics. This is straightforward as a further step of a cut-based analysis 
or of the simple strategy of the " $\eta_{j^{\prime}}$ analysis" $[8,10]([8]$ makes use of three complementary analysis strategies, two of which are multivariate analyses), thanks to the small and calculable correlation between $\eta_{j^{\prime}}$ and $\cos \theta_{\ell}^{*}$, while more care has to be taken in multivariate analyses, where several input observables are correlated with the polarization. A promising way out in this case is the use of orthogonalization techniques, like "deboosting" [23], to ensure that the MVA discriminator used for event selection is uncorrelated with $\cos \theta_{\ell}^{*}$.

Other angular variables can be defined in single top events for the purpose of measuring top quark polarization in a model-independent way (see, e.g., [27]). Several angular observables can be useful, alone or combined, thanks to their sensitivities to the helicity states of the $W$ from top decay, to constrain the parameters of the most general Lagrangian term that one can write for the $t b W$ coupling up to six-dimensional gauge invariant operators [28] (under the approximation $\left.V_{t b}=1\right)$ :

$$
\begin{aligned}
\mathscr{L}_{t b W}= & -\frac{g}{\sqrt{2}} \bar{b}\left[\gamma^{\mu}\left(f_{L} P_{L}+f_{R} P_{R}\right)+\frac{i \sigma^{\mu \nu} q_{v}}{M_{W}}\left(g_{L} P_{L}+g_{R} P_{R}\right)\right] \\
& \times t W_{\mu}^{-}+\text {h.c., }
\end{aligned}
$$

where the SM predicts $f_{L}=1, f_{R}=g_{L}=g_{R}=0$. The D0 collaboration has set constrains on these parameters by means of a neural network analysis combining several angular and kinematical inputs, with different networks trained for nonzero values of each of the anomalous parameters $f_{R}, g_{L}$, and $g_{R}$ [29]. Due to the higher centre-of-mass energy, given that the new physics effects would enter in the production vertex, LHC experiments are expected to be more sensitive to deviations to the SM. The simultaneous measurement of the cross-section and of the parameters of the generalized $t b W$ coupling would help to get rid of the simplifying assumption about $\left|V_{t b}\right|$ implicit in (2).

\section{Deficits and Excesses: A Bottom-Up Approach}

Different beyond-SM models predict different effects in the different production channels [12]. Here, we outline a few examples, reasoning in a bottom-up approach, that is, starting from the possible observations of deficits or excesses to infer which model (including the SM itself) could provide the most natural explanation.

(i) A deficit in all three channels would naturally lead to suspect $\left|V_{t b}\right|<1$ (hence the existence of new quarks); this can be verified by precisely measuring $R_{b} \equiv B R(t \rightarrow W b) / B R(t \rightarrow W q)$ in $t \bar{t}$ events;

(ii) an excess in the $s$-channel, not confirmed in the other two, would induce to suspect a charged resonance (e.g., a right-handed $W^{\prime}$, whose coupling to light fermions would be suppressed by helicity conservation and would, therefore, be visible in the $t \bar{b}$ final state and not in electronic or muonic decays), which would be confirmed by a peak or a peak-dip structure in the $M_{t \bar{b}}$ spectrum;

(iii) an excess in the $t$-channel, not confirmed in the other two, could be due to new interactions causing flavourchanging neutral currents (FCNCs): even with tiny $u t \gamma$ and $u t Z$ couplings, the very large up-quark density at high $x$ in the proton would allow a visible signal to show up; important checks would be the differential measurements of $d \sigma_{t / \bar{t}}^{t \text {-channel }} / d y$ and of the single top polarization;

(iv) an excess in the $t$-channel and in the $t W$ channel, with no deviation from the SM in the polarization of the top quark, and a deficit in the s-channel could be due to large $\left|V_{t d}\right|$ or $\left|V_{t s}\right|$ (nonunitarity of the CKM matrix, hence possible existence of new quarks), to be checked by measuring $d \sigma_{t / \bar{t}}^{t \text {-channel }} / d y$ and $R_{b}$.

Some of these new physics effects in $t$-channel and $t W$ production might be mimicked by discrepancies in the gluon or $b$-quark PDFs at large $x$ and it is, therefore, necessary to be able to rule out this possibility by additional dedicated inputs. Precision measurements of all three production modes will have a deep impact on PDF constraints, with the three channels being complementary to each other and also to $t \bar{t}$ production. For example, $t$-channel and $t W$ cross-sections are sensitive to the $b$-quark PDF and anticorrelated with the $W / Z$ cross-section, while the $s$-channel (essentially a Drell-Yan process) is insensitive to the $b$-quark PDF and can therefore act as a control process, and it is correlated with the $W / Z$ cross-section, like the $t \bar{t}$ cross-section [30]. Moreover, the integrated or differential charge asymmetry in $t$-channel production will provide a very powerful input for constraining PDFs, similar to the $W$ production case, in a region of $x$ very relevant for several other searches. The first measurement of the integrated charge asymmetry $\left(R_{t} \equiv \sigma(t) / \sigma(\bar{t})\right)$ from the ATLAS collaboration [31] at $7 \mathrm{TeV}$ has already disfavoured some PDF sets, as illustrated in Figure 4. Differential distributions of $R_{t}$ as a function of rapidity and transverse momentum of the top quark will provide significant additional discriminating power. Another useful input for constraining PDFs is the measurement of the ratio of single top cross-sections between 7 and $8 \mathrm{TeV}$, as done by the CMS collaboration in the $t$-channel case by taking the ratio of results of the $\eta_{j^{\prime}}$ analysis at the two energies [10].

\section{Exotic Single Top Signatures}

While the " $t$-channel like" signature, as mentioned in Section 4, is sensitive to FCNC couplings of the kinds $u t \gamma$ and $u t Z$, which can also be constrained by looking for the $t \rightarrow Z q$ and $t \rightarrow \gamma q$ decays in pair-produced top quark events (searches for the $t \rightarrow Z q$ decay have been already performed by both LHC collaborations at $7 \mathrm{TeV}[32,33])$, the exotic signature of a "very single top" (i.e., a $2 \rightarrow 1$ partonic reaction producing a top quark, see Figure 5) is by far the most powerful handle to constrain the FCNC couplings of the kind $u t g$, given the overwhelming SM background in the $t \bar{t}$ decays. The CDF and ATLAS collaborations performed 


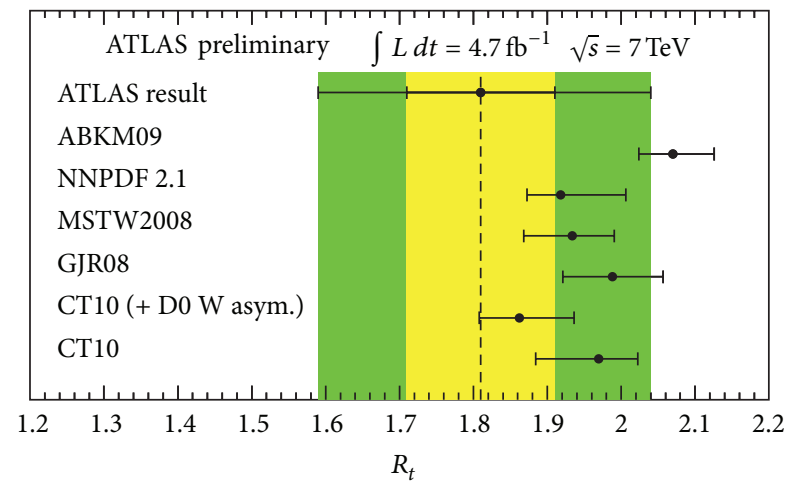

Figure 4: Measured $R_{t}$ value in ATLAS, compared to the values calculated for different NLO PDF sets. The error on the calculated values contains the uncertainty on the renormalisation and factorisation scales. The combined statistical and systematic uncertainty of the measurement is shown in green, while the statistical uncertainty is represented by the yellow error band. Taken from [31].

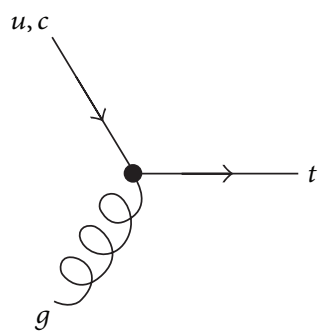

FIGURE 5: Leading order diagram for a "very single top" produced by an anomalous (FCNC) coupling of the kind utg.

a search in the very-single-top final state $[34,35]$, with the tightest bounds to date coming from ATLAS at $7 \mathrm{TeV}$ :

$$
\begin{aligned}
\frac{K_{t g u}}{\Lambda} & <0.0069 \mathrm{TeV}^{-1} \\
\frac{K_{t g c}}{\Lambda} & <0.016 \mathrm{TeV}^{-1}
\end{aligned}
$$

The D0 collaboration chose a different strategy, based on the SM-like signature of a single top quark in association with a light quark [36] (same signature as $t$-channel production in the SM). With a similar integrated luminosity as $\operatorname{CDF}\left(2.3 \mathrm{fb}^{-1}\right.$ versus $2.2 \mathrm{fb}^{-1}$ for $\left.\mathrm{CDF}\right)$, they obtained more stringent limits, demonstrating that the "top + jet" signature (mostly initiated by quark-quark collisions) is more sensitive than the "very-single-top" signature in the Tevatron conditions. Nevertheless, at the LHC energies the gluon flux is much more intense than at Tevatron in comparison to the quark flux; therefore, the balance is likely in favour of the "very single top" (A proper comparison, beyond the scope of this paper, would demand to take into account any effect susceptible to increase the jet multiplicity of the event, like initial state radiation and pileup).

New physics can produce a single top quark in association with invisible particles, hence large missing transverse energy (this signature is known in the literature as "monotops") [37], or a single top in association with a $Z$ boson, which in turn can give the monotop signature (when the $Z$ boson decays into a neutrino pair) or a spectacular final state of three leptons, a $b$ jet and large transverse missing energy. No experimental collaboration published a search for these final states yet. It can be remarked, in the context of this volume dedicated to new heavy quarks, that both signatures can be originated by the production of heavy vector-like partners of the top quark, which would have the FCNC decay $t^{\prime} \rightarrow t Z$ [38]. Given the very tight limits on a sequential fourth generation with SM-like couplings, the exploration of these alternative new quark scenarios becomes even more interesting, and single production is kinematically favoured over pair production, despite the larger QCD coupling, when the quark is very heavy.

\section{Conclusions}

The single top final states are an important place to look for indirect evidences of new heavy quarks, and the measurements of the properties of single top quarks are crucial to gain sensitivity to non-SM interactions and to get some insight into the kind of new physics at hand in case of deviations from the SM hypothesis.

\section{Acknowledgments}

The author wishes to thank Paris Sphicas and George Hou for their suggestions, and the Estonian Academy of Science for supporting this work with the Mobilitas Top Researcher Grant MTT59.

\section{References}

[1] T. Aaltonen, J. Adelman, T. Akimoto et al., "First observation of electroweak single top quark production," Physical Review Letters, vol. 103, Article ID 092002, 9 pages, 2009.

[2] V. M. Abazov, B. Abbott, M. Abolins et al., "Observation of single top-quark production," Physical Review Letters, vol. 103, Article ID 092001, 7 pages, 2009.

[3] G. Aad, B. Abbott, J. Abdallah et al., "The ATLAS experiment at the CERN LHC," Journal of Instrumentation, vol. 3, Article ID S08003, 2008.

[4] S. Chatrchyan, G. Hmayakyan, V. Khachatryan et al., "The CMS experiment at the CERN LHC," Journal of Instrumentation, vol. 3, no. 8, Article ID S08004, 2008.

[5] G. Aad, B. Abbott, J. Abdallah et al., "Evidence for the associated production of a $W$ boson and a top quark in ATLAS at $\sqrt{s}=$ 7 TeV,' Physics Letters B, vol. 716, no. 1, pp. 142-159, 2012.

[6] S. Chatrchyan, G. Hmayakyan, V. Khachatryan et al., "Evidence for associated production of a single top quark and $W$ boson in pp collisions at 7 TeV," Physical Review Letters, vol. 110, no. 2, Article ID 022003, 16 pages, 2013.

[7] G. Aad, B. Abbott, J. Abdallah et al., "Measurement of the $t$-channel single top-quark production cross section in $p p$ collisions at $\sqrt{s}=7 \mathrm{TeV}$ with the ATLAS detector," Physics Letters B, vol. 717, no. 4-5, pp. 330-350, 2012.

[8] S. Chatrchyan, G. Hmayakyan, V. Khachatryan et al., "Measurement of the single-top-quark $t$-channel cross section in $p p$ 
collisions at $\sqrt{s}=7 \mathrm{TeV}$,' Journal of High Energy Physics, vol. 1212, article 035, 2012.

[9] G. Cataldi, A. dos Anjos, W. Wiedenmann et al., "Measurement of $t$-channel single top-quark production in $p p$ collisions at $\sqrt{s}$ $=8 \mathrm{TeV}$ with the ATLAS detector," ATLAS-CONF-2012-132.

[10] S. Chatrchyan, G. Hmayakyan, V. Khachatryan et al., "Measurement of the single-top $t$-channel cross section in $p p$ collisions at centre-of-mass energy of $8 \mathrm{TeV}$," CMS-TOP-12-011.

[11] V. M. Abazov, B. Abbott, M. Abolins et al., "Precision measurement of the ratio $B(t \rightarrow W b) / B(t \rightarrow W q)$ and extraction of $V_{t b}$, Physical Review Letters, vol. 107, Article ID 121802, 2011.

[12] T. M. P. Tait and C. P. Yuan, "Single top quark production as a window to physics beyond the standard model," Physical Review $D$, vol. 63, no. 1, Article ID 014018, 5 pages, 2001.

[13] J. Alwall, R. Frederix, J. M. Gérard et al., "Is $V_{t b} \approx 1$ ?" European Physical Journal C, vol. 49, no. 3, pp. 791-801, 2007.

[14] H. Lacker, A. Menzel, F. Spettel et al., "Model-independent extraction of $\left|V_{t q}\right|$ matrix elements from top-quark measurements at hadron colliders," European Physical Journal C, vol. 72, article 2048.

[15] N. Kidonakis, "Differential and total cross sections for top pair and single top production," http://arxiv.org/abs/1205.3453v1.

[16] T. Aaltonen, J. Adelman, T. Akimoto et al., "Measurement of single top quark production in $7.5 \mathrm{fb}^{-1}$ of CDF Data Using Neural Networks," CDF conf. note 10793.

[17] V. M. Abazov, B. Abbott, M. Abolins et al., "Model-independent measurement of $t$-channel single top quark production in $p p$ collisions at $\sqrt{s}=1.96 \mathrm{TeV}$," Physics Letters $B$, vol. 705, no. 4, pp. 313-319, 2011.

[18] Tevatron Electroweak Working Group, "Combination of CDF and D0 measurements of the single top production cross section," http://arxiv.org/abs/0908.2171.

[19] V. M. Abazov, B. Abbott, M. Abolins et al., "Measurements of single top quark production cross sections and $\left|V_{t b}\right|$ in $p p$ collisions at $\sqrt{s}=1.96 \mathrm{TeV}$," Physical Review D, vol. 84, Article ID 112001, 2011.

[20] M. Jezabek and J. H. Kühn, "V-A tests through leptons from polarised top quarks," Physics Letters B, vol. 329, no. 2-3, pp. 317324, 1994.

[21] G. Mahlon and S. Parke, "Single top quark production at the LHC: understanding spin," Physics Letters B, vol. 476, no. 3-4, pp. 323-330, 2000.

[22] S. Chatrchyan, G. Hmayakyan, V. Khachatryan et al., "Measurement of the $t$-channel single top quark production cross section in $p p$ collisions at $\sqrt{s}=7 \mathrm{TeV}$," Physical Review Letters, vol. 107, Article ID 091802, 2011.

[23] D. Martschei, M. Feindt, S. Honc, and J. Wagner-Kuhr, "Advanced event reweighting using multivariate analysis," Journal of Physics, vol. 368, Article ID 012028, 2012.

[24] J. M. Campbell, R. Frederix, F. Maltoni, and F. Tramontano, "NLO predictions for $t$-channel production of single top and fourth generation quarks at hadron colliders," Journal of High Energy Physics, vol. 2009, no. 10 article 042, 2009.

[25] N. Kidonakis, "Next-to-next-to-leading-order collinear and soft gluon corrections for $t$-channel single top quark production," Physical Review D, vol. 83, no. 9, Article ID 091503, 2011.

[26] J. M. Campbell, J. W. Huston, and W. J. Stirling, "Hard interactions of quarks and gluons: a primer for LHC physics," Reports on Progress in Physics, vol. 70, no. 1, article 89, 2007.

[27] J. A. Aguilar-Saavedra and R. V. Herrero-Hahn, "Modelindependent measurement of the top quark polarisation," http://arxiv.org/abs/1208.6006.
[28] J. A. Aguilar-Saavedra, "A minimal set of top anomalous couplings," Nuclear Physics B, vol. 812, no. 1-2, pp. 181-204, 2009.

[29] V. M. Abazov, B. Abbott, M. Abolins et al., "Combination of searches for anomalous top quark couplings with $5.4 \mathrm{fb}^{-1}$ of $p \bar{p}$ collisions," Physics Letters B, vol. 713, pp. 165-171, 2012.

[30] A. Guffanti and J. Rojo, "Top production at the LHC: the impact of PDF uncertainties and correlations," http:// arxiv.org/abs/1008.4671.

[31] G. Cataldi, A. dos Anjos, W. Wiedenmann et al., "Measurement of the $t$-channel single top-quark and top-antiquark crosssections and their ratio in $p p$ collisions at $\sqrt{s}=7 \mathrm{TeV}$," ATLASCONF-2012-056.

[32] G. Cataldi, A. dos Anjos, W. Wiedenmann et al., "A search for flavour changing neutral currents in top-quark decays in $p p$ collision data collected with the ATLAS detector at $\sqrt{s}=7 \mathrm{TeV}$," http://arxiv.org/abs/1206.0257.

[33] S. Chatrchyan, G. Hmayakyan, V. Khachatryan et al., "Search for flavour changing neutral currents in top quark decays in $p p$ collisions at $7 \mathrm{TeV}$," http://arxiv.org/abs/1208.0957.

[34] T. Aaltonen, J. Adelman, T. Akimoto et al., "Search for topquark production via flavor-changing neutral currents in $W+1$ jet events at CDF," Physical Review Letters, vol. 102, no. 15, Article ID 151801, 7 pages, 2009.

[35] G. Cataldi, A. dos Anjos, W. Wiedenmann et al., "Search for FCNC single top-quark production at $\sqrt{s}=7 \mathrm{TeV}$ with the ATLAS detector," vol. 712, no. 4-5, pp. 351-369, 2012.

[36] V. M. Abazov, B. Abbott, M. Abolins et al., "Search for flavor changing neutral currents via quark-gluon couplings in single top quark production using $2.3 \mathrm{fb}^{-1}$ of $p \bar{p}$ collisions," Physics Letters B, vol. 693, no. 2, pp. 81-87, 2010.

[37] J. Andrea, B. Fuks, and F. Maltoni, "Monotops at the LHC," http://arxiv.org/abs/1106.6199.

[38] G. Cacciapaglia, A. Deandrea, N. Gaur, D. Harada, Y. Okada, and L. Panizzi, "Heavy Vector-like top partners at the LHC and avourconstraints," http://arxiv.org/abs/1108.6329. 

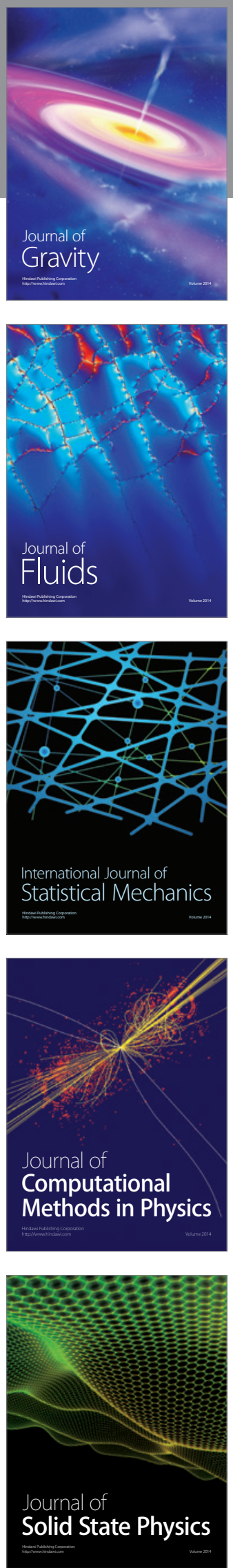

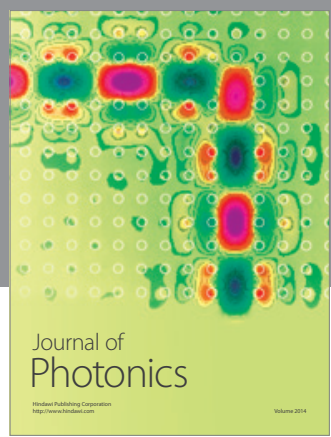

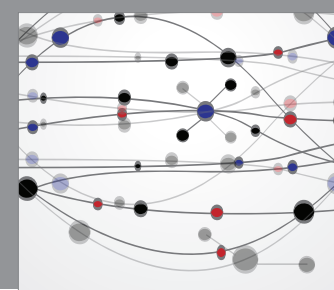

The Scientific World Journal

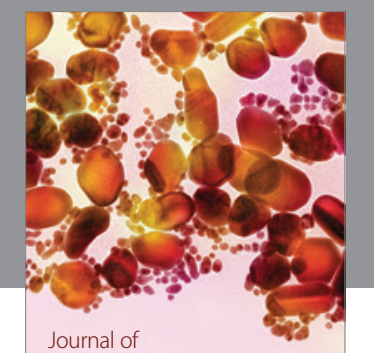

Soft Matter
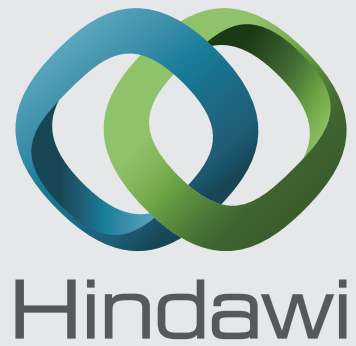

Submit your manuscripts at

http://www.hindawi.com
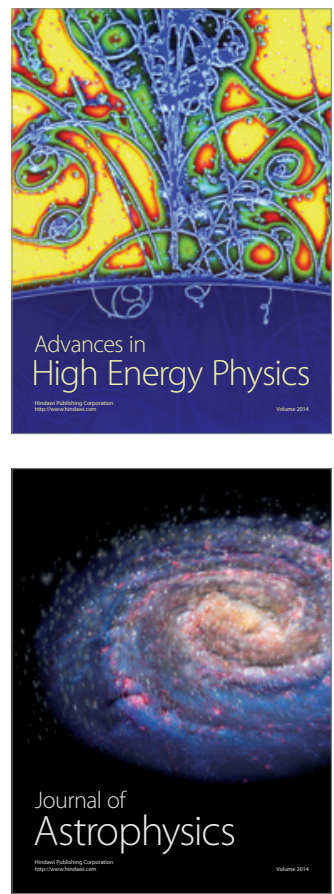
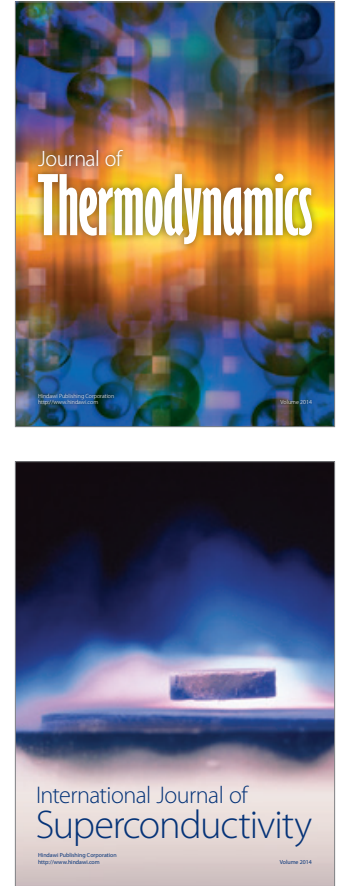
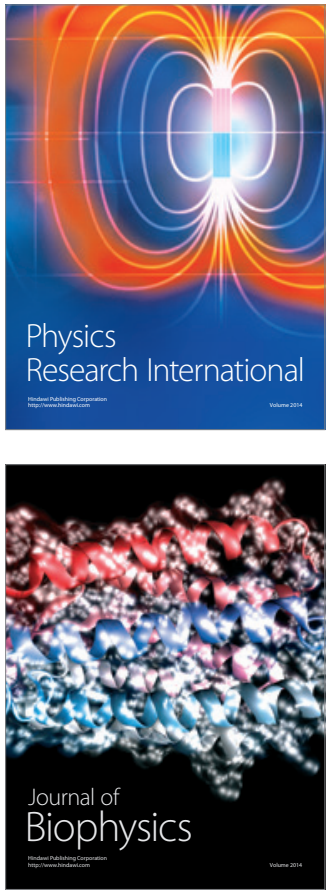
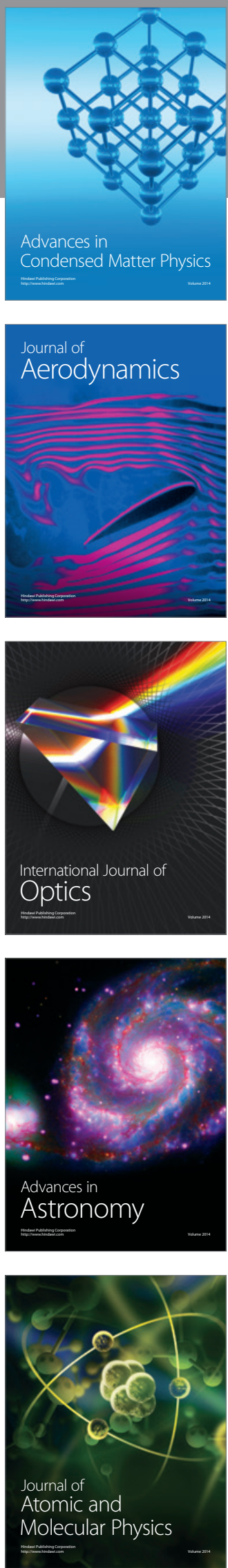